Case Report

\title{
Medial Radial Head Dislocation Associated with a Proximal Olecranon Fracture: A Bado Type V?
}

\author{
Neil Segaren, ${ }^{1}$ Hani B. Abdul-Jabar, ${ }^{1}$ Nicholas Segaren, ${ }^{2}$ and Matthew Barry ${ }^{3}$ \\ ${ }^{1}$ The Catterall Unit, Royal National Orthopaedic Hospital, Brockley Hill, Stanmore, Middlesex HA7 4LP, UK \\ ${ }^{2}$ School of Public Health, Harvard University, 677 Huntington Avenue, Boston, MA 02115 6018, USA \\ ${ }^{3}$ The Royal London Hospital, Whitechapel Road, London E1 1BB, UK \\ Correspondence should be addressed to Hani B. Abdul-Jabar; hba999@gmail.com
}

Received 7 January 2014; Accepted 9 March 2014; Published 17 March 2014

Academic Editors: A. Cho and G. Rallis

Copyright (C) 2014 Neil Segaren et al. This is an open access article distributed under the Creative Commons Attribution License, which permits unrestricted use, distribution, and reproduction in any medium, provided the original work is properly cited.

\begin{abstract}
The Monteggia fracture is relatively rare. We present an unreported configuration of a traumatic olecranon fracture with a concomitant medial radial head dislocation in a 3-year-old male. This injury was initially missed and required a subsequent operative intervention. Following surgery, there was evident fracture union, articular congruency, and a full functional recovery. Medial radial head dislocation is not accounted for in the Bado (1967) classification of the Monteggia lesion and hence we propose the addition of a Bado V category.
\end{abstract}

\section{Introduction}

The Monteggia fracture is relatively rare in the paediatric population and has been classified by Bado [1] into four variants. We present the case of a three-year-old boy who sustained an olecranon fracture with a concomitant medial dislocation of the radial head. We propose the addition of a 5th variant to the Bado classification to encompass medial and anteromedial radial head dislocations.

\section{Case Report}

The patient's guardian gave informed consent prior to the child being included into the study. This study was authorized by the local ethical committee and was performed in accordance with the Ethical standards of the 1964 Declaration of Helsinki as revised in 2000.

A three-year-old boy fell whilst running in a playground. He presented to the emergency department at his local hospital and was complaining of a painful elbow. On examination, his elbow was noted to be extremely swollen and has adapted a flexed posture with maximal apprehension and reluctance at any attempt to mobilise its joints in all directions. Plain radiographs were obtained (Figures 1 and 2) which confirmed an olecranon fracture in the presence of a radial head dislocation. The radial head dislocation was not initially recognised by the assessing casualty doctor. The elbow was immobilised in an above elbow back slab and the patient was discharged home with a planned follow-up in fracture clinic where he was seen by an orthopaedic registrar 13 days after the injury. Repeat radiographs were obtained (Figures 3 and 4) and the medial radial head dislocation was confirmed.

Due to the nature of this unusual presentation; the child was referred to the regional tertiary paediatric orthopaedic centre for further assessment in view of a possible need for open reduction and operative intervention. Fifteen days after injury, the child was taken to the operating theatre; initial examination under anaesthesia revealed flexion-extension arc from 0 to 130 degrees and 5 degrees each of supination and pronation.

A direct posterior approach was utilised to openly expose the olecranon fracture which showed signs of early callus deposition. The olecranon fracture was mobilised fully beyond its assumptive initial fracture position; allowing adequate visualisation of the radial head, which had surprisingly dislocated medially. The radial head was reduced under direct vision, the ulnar fracture was then anatomically 


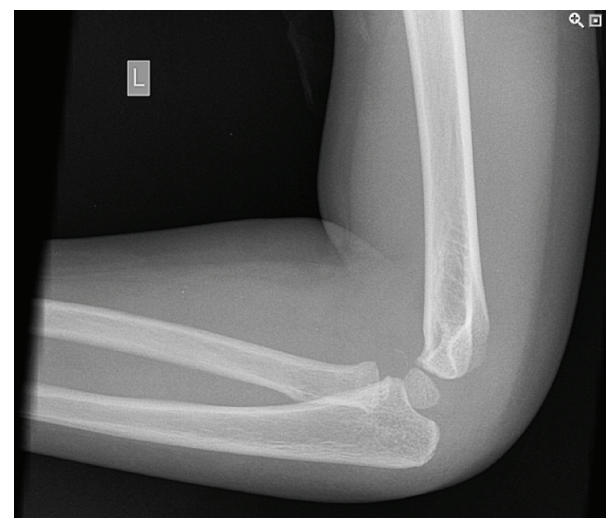

FIGURE 1: Lateral radiograph of three-year-old boy showing olecranon fracture.

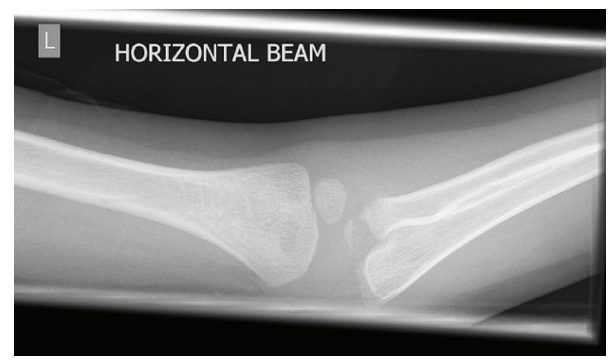

FIGURE 2: Anterioposterior radiograph: note the bony fragment and the medial radial head dislocation.

reduced and stabilised with two crossed Kirschner wires. Examination following articular congruency and fracture fixation demonstrated a full range of anatomical supinationpronation arc.

Postoperatively, the arm was immobilised for four weeks in an above elbow plaster of Paris. The wires were removed following plaster disposal and the patient was encouraged to actively mobilise his elbow as comfort allowed. At his last follow-up 8 months after open reduction and internal fixation, the child had a pain-free elbow with a full arc range of movement. Radiographs (Figures 5 and 6) demonstrated full union of the olecranon fracture and elbow joint articular congruency.

\section{Discussion}

Monteggia fractures are uncommon and account for about $0.7 \%$ of elbow fractures and dislocations [2] and $7 \%$ of fractures of the radius and ulna [3]. Bado [1] classified the Monteggia lesion into four types (Table 1), depending on the direction of the radial head dislocation. Traumatic medial dislocation of the radial head associated with a proximal olecranon fracture is a rare injury and to our knowledge has not been reported within the scientific literature to date. Anteromedial dislocation of the radial head has been described in low energy trauma in association with an undisplaced olecranon fracture [4] and with [5] or without [6]

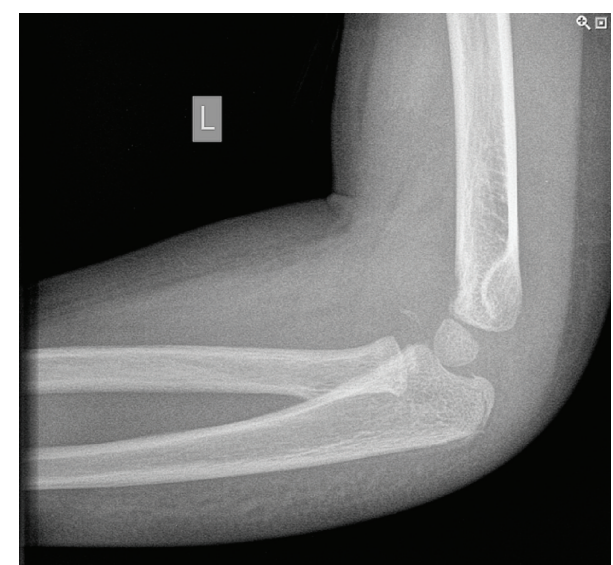

FIgURE 3: Repeat lateral radiograph of the same elbow twelve days later. The olecranon fracture is more defined.

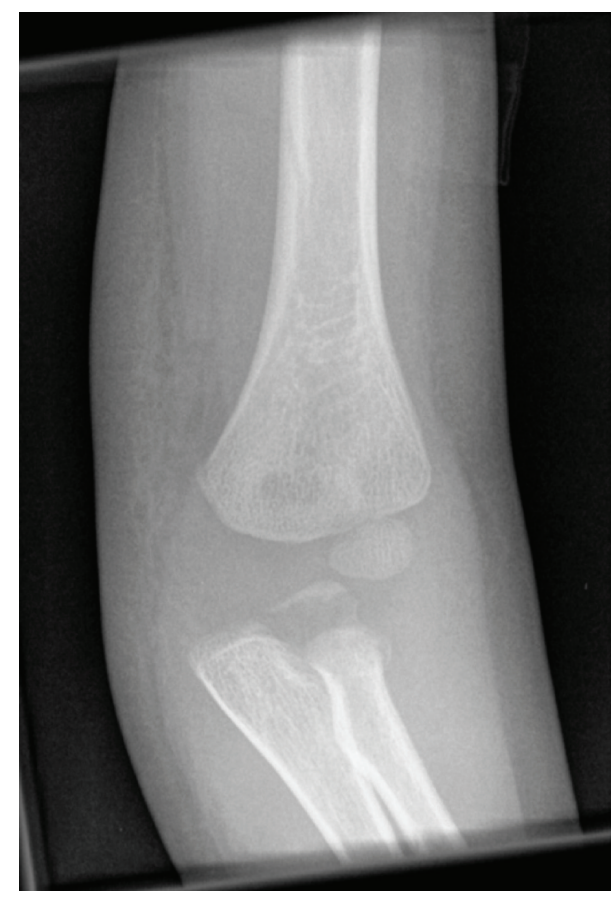

FIGURE 4: Anterioposterior radiograph of the same elbow twelve days after injury. Bony fragment still visible.

radial head fracture, and in isolation as a consequence of birth injury [7].

Due to the unique fracture configuration, the radial head dislocation was not identified for 15 days. The senior author felt that an open reduction offered the greatest chance of an optimal outcome.

In an isolated anteromedial radial head dislocation, closed reduction alone is often unsuccessful due to soft tissue interposition from the annular ligament [8], biceps tendon $[5,6]$, median nerve [9], radial nerve [10], or joint capsule $[4,11]$. The direct posterior approach used in this case provided a safe operative window allowing full visualisation 
TABle 1: Bado [1] classification of the Monteggia lesion with the proposed type V category.

\begin{tabular}{ll}
\hline Bado Type & Description \\
\hline Type I (60\%) & Anterior dislocation of the radial head. Fracture of the ulna with anterior angulation. \\
Type II $(15 \%)$ & Posterior or postero-lateral dislocation of the radial head. Fracture of the ulna with posterior angulation. \\
Type III $(20 \%)$ & Lateral or antero-lateral dislocation of the radial head. Fracture of ulna metaphysis. \\
Type IV $(<5 \%)$ & Anterior dislocation of the radial head with fracture of the proximal third of the radius and fracture of ulna at \\
Type V (proposed) & Medial or antero-medial dislocation of the radial head with fracture of the proximal ulna. \\
\hline
\end{tabular}

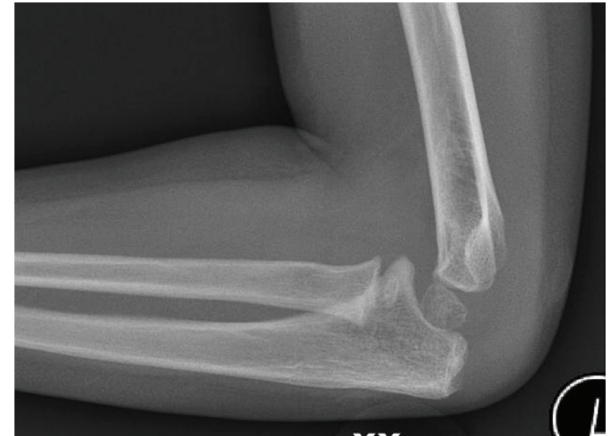

FigURE 5: Lateral radiograph four months postoperatively. Note the union of the olecranon fracture and the satisfactory position of the radiocapitellar joint.

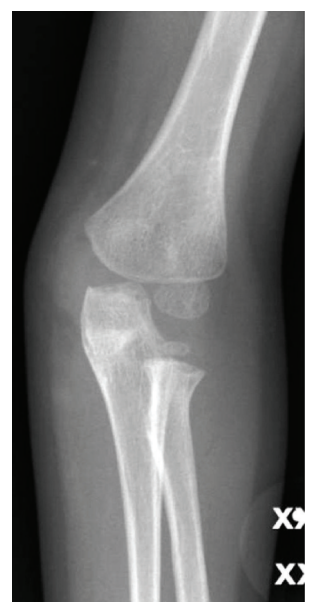

FIGURE 6: Anteroposterior radiograph four months postoperatively. Note the union of the olecranon fracture and the satisfactory position of the radiocapitellar joint.

and subsequent reduction of the dislocated radial head and anatomical fixation of the associated olecranon fracture.

In summary, we report a traumatic medial radial head dislocation associated with a displaced olecranon fracture. We do not believe that this pattern has been reported in the literature although four cases of anteromedial dislocation have been reported [4-7]. Bado [1] stated that any dislocation (of the radial head) found with an ulna fracture constitutes the anatomical-clinical picture of the lesion discussed by
Monteggia. The current classification of the Monteggia lesion does not account for medial or anteromedial radial head dislocation; hence the authors propose the addition of a Bado type V (Table 1).

\section{Conflict of Interests}

The authors declare that there is no conflict of interests regarding the publication of this paper.

\section{Acknowledgment}

The work is attributed to Royal London Hospital, Whitechapel, London, UK.

\section{References}

[1] J. L. Bado, “The monteggia lesion," Clinical Orthopaedics and Related Research, vol. 50, pp. 71-86, 1967.

[2] P. D. Wilson, "Fractures and dislocations in the region of the elbow," The Journal of Surgery, Gynecology and Obstetrics, vol. 56, pp. 335-339, 1933.

[3] E. G. Edwards, “The posterior Monteggia fracture," The American Surgeon, vol. 18, no. 3, pp. 323-327, 1952.

[4] K. Takase and J. Mizuochi, "Irreducible dislocation of the radial head with undisplaced olecranon fracture in a child: a case report," Journal of Pediatric Orthopaedics Part B, vol. 20, no. 5, pp. 345-348, 2011.

[5] Y. Yoshihara, K. Shiraishi, and K. Imamura, "Irreducible anteromedial dislocation of the radial head caused by biceps tendon clinging around the radial neck," Journal of Trauma-Injury, Infection and Critical Care, vol. 53, no. 5, pp. 984-986, 2002.

[6] K. M. Veenstra and J. W. van der Eyken, "Irreducible anteromedial dislocation of the radius: a case of biceps tendon interposition," Acta Orthopaedica Scandinavica, vol. 64, no. 2, pp. 224-225, 1993.

[7] O. Bayne and M. Rang, "Medial dislocation of the radial heal following breech delivery: a case report and review of the literature," Journal of Pediatric Orthopaedics, vol. 4, no. 4, pp. 485-487, 1984.

[8] K. C. Kong, "Irreducible isolated dislocation of the radial head in a skeletally mature teenager. A case report," Archives of Orthopaedic and Trauma Surgery, vol. 112, no. 6, pp. 304-305, 1993.

[9] J. A. S. Watson, "Irreducible monteggia fracture: beware nerve entrapment," Injury, vol. 25, no. 5, pp. 325-327, 1994. 
[10] W. B. Rodgers, P. M. Waters, and J. E. Hall, "Chronic Monteggia lesions in children. Complications and results of reconstruction," Journal of Bone and Joint Surgery A, vol. 78, no. 9, pp. 13221329, 1996.

[11] R. J. Neviaser and G. W. LeFevre, "Irreducible isolated dislocation of the radial head. A case report," Clinical Orthopaedics and Related Research, vol. 80, pp. 72-74, 1971. 


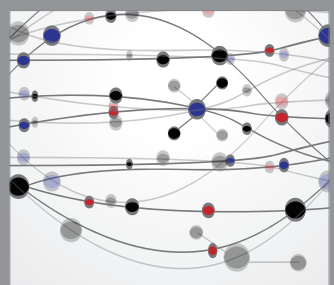

The Scientific World Journal
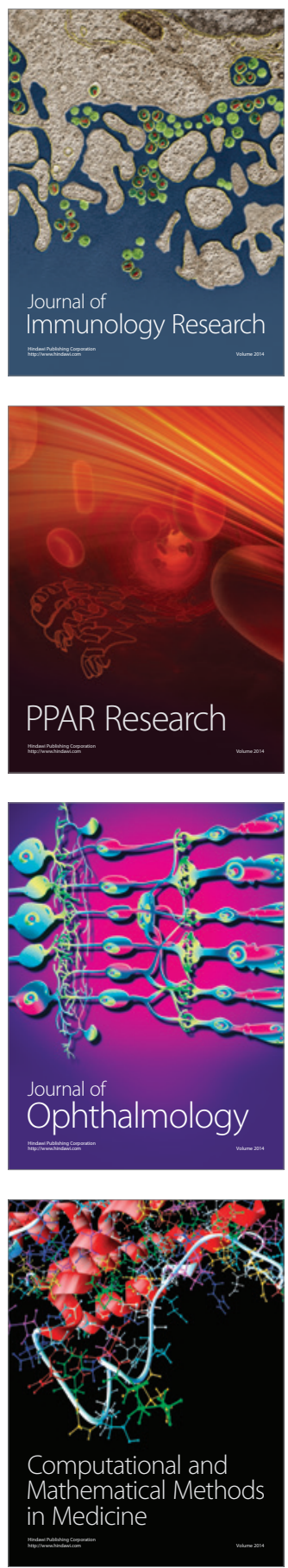

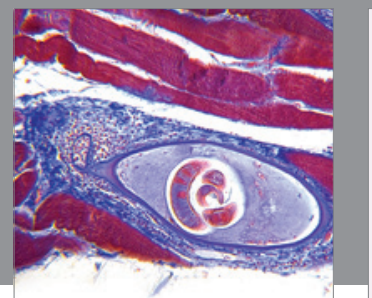

Gastroenterology

Research and Practice
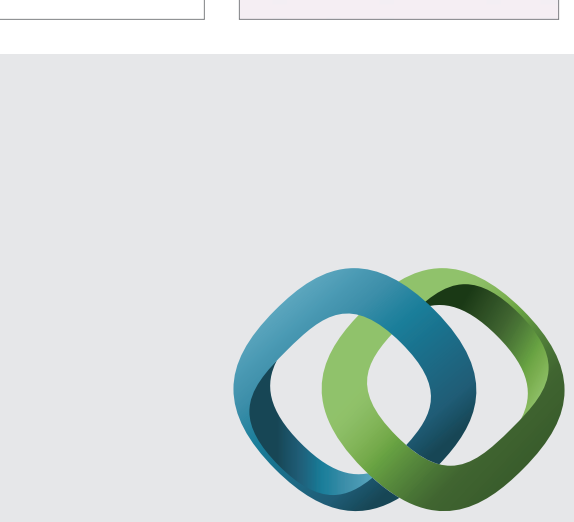

\section{Hindawi}

Submit your manuscripts at

http://www.hindawi.com
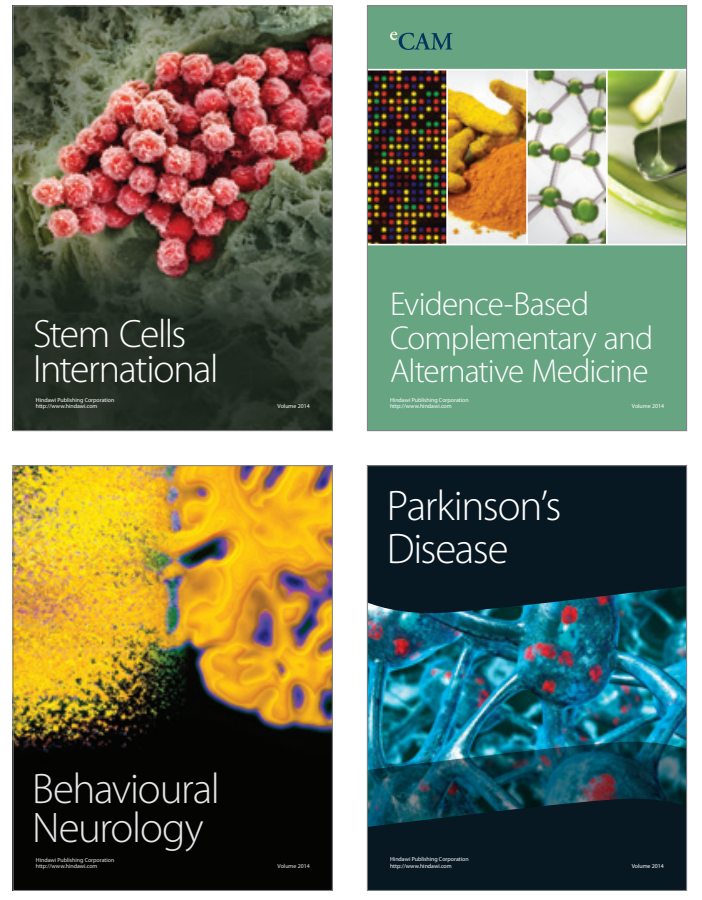
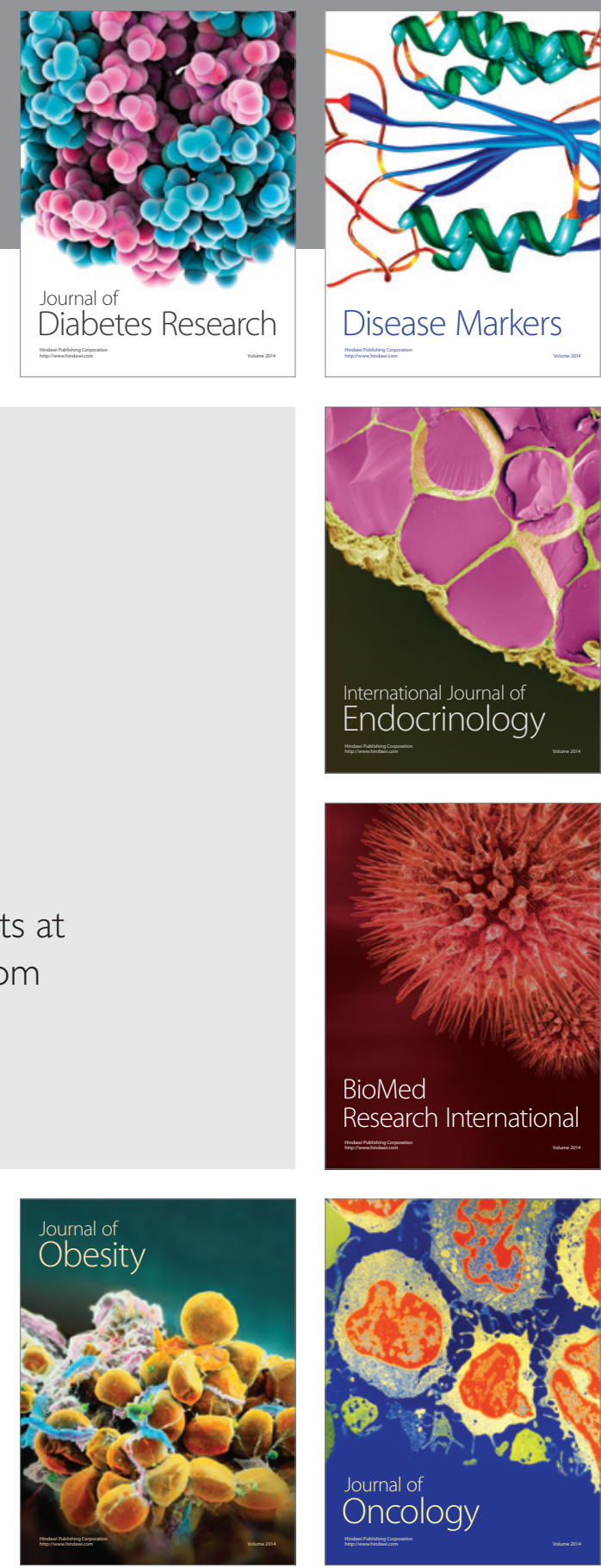

Disease Markers
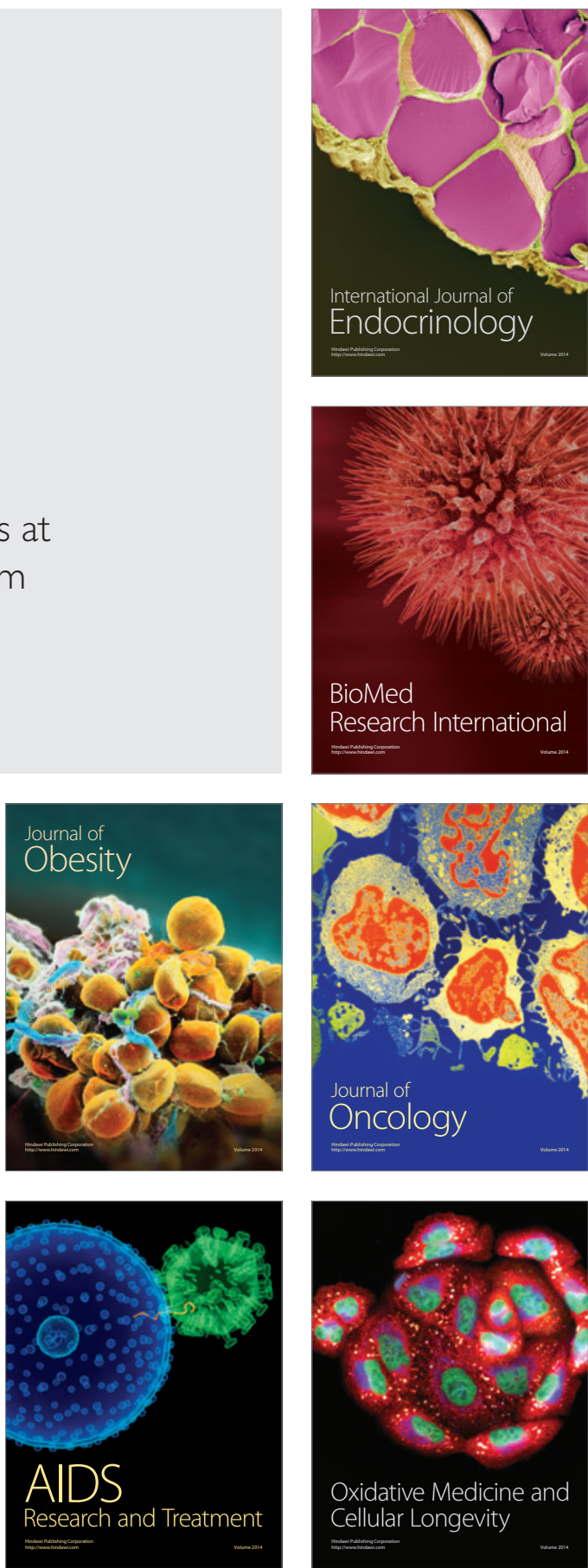